\title{
High-efficiency control of spin-wave propagation in ultra-thin yttrium iron garnet by the spin-orbit torque
}

Cite as: Appl. Phys. Lett. 108, 172406 (2016); https://doi.org/10.1063/1.4948252

Submitted: 01 March 2016 . Accepted: 15 April 2016 . Published Online: 27 April 2016

M. Evelt, V. E. Demidov, V. Bessonov, S. O. Demokritov, J. L. Prieto, M. Muñoz, J. Ben Youssef (D), V. V. Naletov, C. de Loubens, O. Klein, M. Collet (D), K. Garcia-Hernandez (D), P. Bortolotti, V. Cros, and A. Anane
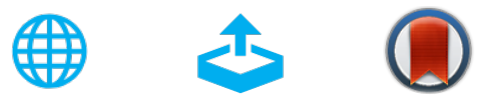

View Online

\section{ARTICLES YOU MAY BE INTERESTED IN}

Spin-wave propagation in ultra-thin YIG based waveguides

Applied Physics Letters 110, 092408 (2017); https://doi.org/10.1063/1.4976708

The design and verification of MuMax3

AIP Advances 4, 107133 (2014); https://doi.org/10.1063/1.4899186

Experimental prototype of a spin-wave majority gate

Applied Physics Letters 110, 152401 (2017); https://doi.org/10.1063/1.4979840 


\title{
High-efficiency control of spin-wave propagation in ultra-thin yttrium iron garnet by the spin-orbit torque
}

\author{
M. Evelt, ${ }^{1}$ V. E. Demidov, ${ }^{1, a)}$ V. Bessonov, ${ }^{2}$ S. O. Demokritov, ${ }^{1,2}$ J. L. Prieto, ${ }^{3}$ M. Muñoz, ${ }^{4}$ \\ J. Ben Youssef, ${ }^{5}$ V. V. Naletov, ${ }^{6,7}$ G. de Loubens, ${ }^{6}$ O. Klein, ${ }^{8}$ M. Collet, ${ }^{9}$ \\ K. Garcia-Hernandez, ${ }^{9}$ P. Bortolotti, ${ }^{9}$ V. Cros, ${ }^{9}$ and A. Anane ${ }^{9}$ \\ ${ }^{1}$ Institute for Applied Physics and Center for Nanotechnology, University of Muenster, 48149 Muenster, \\ Germany \\ ${ }^{2}$ M.N. Miheev Institute of Metal Physics of Ural Branch of Russian Academy of Sciences, \\ Yekaterinburg 620041, Russia \\ ${ }^{3}$ Instituto de Sistemas Optoelectrónicos y Microtecnologa (UPM), Ciudad Universitaria, Madrid 28040, Spain \\ ${ }^{4}$ IMM-Instituto de Microelectrónica de Madrid (CNM-CSIC), PTM, E-28760 Tres Cantos, Madrid, Spain \\ ${ }^{5}$ Laboratoire de Magnétisme de Bretagne CNRS, Université de Bretagne Occidentale, 29285 Brest, France \\ ${ }^{6}$ Service de Physique de l' État Condensé, CEA, CNRS, Université Paris-Saclay, CEA Saclay, \\ 91191 Gif-sur-Yvette, France \\ ${ }^{7}$ Institute of Physics, Kazan Federal University, Kazan 420008, Russian Federation \\ ${ }^{8}$ INAC-SPINTEC, CEA/CNRS and Univ. Grenoble Alpes, 38000 Grenoble, France \\ ${ }^{9}$ Unité Mixte de Physique CNRS, Thales, Univ. Paris Sud, Université Paris-Saclay, 91767 Palaiseau, France
}

(Received 1 March 2016; accepted 15 April 2016; published online 27 April 2016)

\begin{abstract}
We study experimentally with submicrometer spatial resolution the propagation of spin waves in microscopic waveguides based on the nanometer-thick yttrium iron garnet and Pt layers. We demonstrate that by using the spin-orbit torque, the propagation length of the spin waves in such systems can be increased by nearly a factor of 10 , which corresponds to the increase in the spin-wave intensity at the output of a $10 \mu \mathrm{m}$ long transmission line by three orders of magnitude. We also show that, in the regime, where the magnetic damping is completely compensated by the spin-orbit torque, the spin-wave amplification is suppressed by the nonlinear scattering of the coherent spin waves from current-induced excitations. Published by AIP Publishing.

[http://dx.doi.org/10.1063/1.4948252]
\end{abstract}

The spin-orbit torque (SOT) produced by the spin Hall effect $^{1,2}$ (SHE) or the Rashba-Edelstein effect ${ }^{3,4}$ is currently considered as a promising mechanism for the magnetization switching ${ }^{5-9}$ and the excitation and control of the magnetization dynamics ${ }^{10-16}$ in the magnetic nano-systems. The significant advantage of the SOT for the emerging field of magnonics ${ }^{17-19}$ utilizing spin waves as a signal carrier in nano-circuits is the possibility to exert a spin-transfer torque over extended areas. In contrast to the spin transfer torque produced by the spin-polarized electric currents ${ }^{20,21}$ or by the non-local spin injection, ${ }^{22,23}$ the SOT provides an opportunity not only to excite local magnetization oscillations but also to control the propagation length of spin waves. Although the idea of the propagation-length control is straightforward, the efficient modulation of the spin-wave propagation characteristics by the spin torque has not been achieved so far. Even in all-metallic systems, where the SOT is known to be very efficient, the maximum achieved increase in the propagation length was less than a factor of $2 .^{15,16}$

An additional important advantage of SOT is the possibility to implement the spin-torque devices based on the insulating magnetic materials, ${ }^{24}$ such as yttrium iron garnet (YIG), which possess significantly smaller magnetic damping compared to the metallic ferromagnets. ${ }^{25}$ In recent years, the applicability of this material for spin-torque devices was limited by the large micrometer-range thickness of the high-

\footnotetext{
${ }^{\text {a) }}$ Author to whom correspondence should be addressed. Electronic mail: demidov@uni-muenster.de
}

quality YIG films. Since SOT is the interfacial phenomenon, the large thickness of the active magnetic layer caused a low efficiency of devices and resulted in the moderate effect of SOT on the propagation characteristics of spin waves. ${ }^{26,27}$ Only very recently, with the developments in preparation of nanometer-thick low-damping YIG films, ${ }^{28-33}$ the implementation of the insulator-based spin-torque devices became practically feasible. ${ }^{33,34}$

In this letter, we study the propagation of spin waves in 20-nm thick microscopic YIG waveguides subjected to SOT generated by SHE in an adjacent Pt film. We demonstrate that this system provides an opportunity of highly efficient control of the propagation characteristics of spin waves. In particular, we achieve nearly a tenfold increase in the propagation length. The observed variation of the propagation length is in perfect agreement with the simple theory predicting a linear dependence of the effective damping on the electrical current inducing the spin-orbit torque. Additionally, the high efficiency of our system allowed us to study the regime, where the damping is overcompensated by SOT and the true amplification of spin waves is expected. We show that, in this regime, the spin system of the YIG film is strongly overdriven, which results in the suppression of the spin-wave amplification.

Figure 1 shows the schematic of the test devices. They are based on a $20 \mathrm{~nm}$ thick YIG film grown by the pulsed laser deposition on the gadolinium gallium garnet (GGG) (111) substrate. ${ }^{29}$ The film is covered by an $8 \mathrm{~nm}$ thick layer of Pt deposited using dc magnetron sputtering, and the 


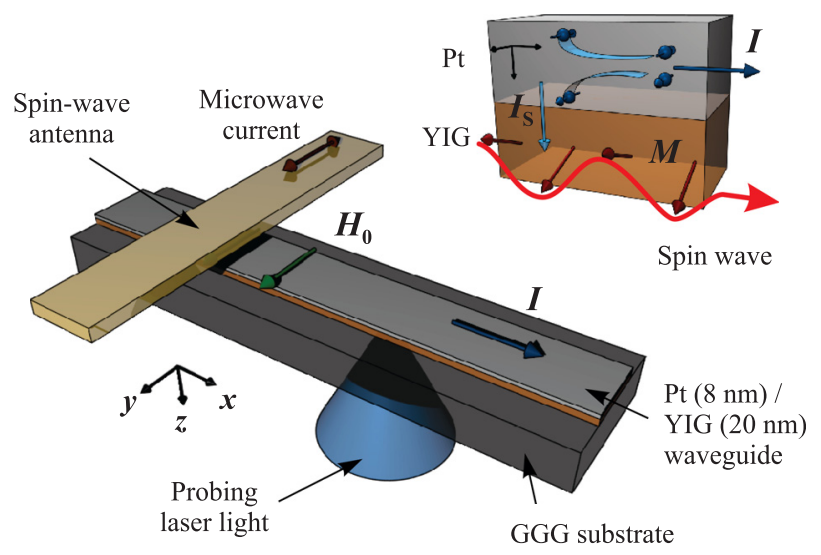

FIG. 1. Schematic of the experiment. Inset illustrates the generation of the pure spin current by the spin-Hall effect.

YIG/Pt bilayer is patterned by e-beam lithography into a stripe waveguide with the width of $1 \mu \mathrm{m}$. The system is insulated by a $300 \mathrm{~nm}$ thick $\mathrm{SiO}_{2}$ layer, and a broadband $3 \mu \mathrm{m}$ wide microwave antenna made of $250 \mathrm{~nm}$ thick $\mathrm{Au}$ is defined on top of the system by the optical lithography. The waveguide is magnetized by the static magnetic field $H_{0}=1000$ Oe applied in its plane perpendicular to the long axis. A dc electrical current $I$ flowing in the plane of the $\mathrm{Pt}$ film is converted by the SHE into the transverse spin accumulation (see inset in Fig. 1). The associated pure spin current $I_{\mathrm{S}}$ is injected into the YIG film resulting in a spintransfer torque on its magnetization $M$. Depending on the relative orientation of the current and the static magnetic field, the SOT either compensates or enhances the effective magnetic damping in the YIG film.

The magnetic dynamics in YIG is analyzed by using the micro-focus Brillouin light scattering (BLS) spectroscopy. ${ }^{35}$ The probing laser light generated by a single-frequency laser is sent through the GGG substrate and is focused by a specially corrected microscope objective lens into a diffractionlimited spot on the YIG/Pt surface. Due to the interaction of the light with the magnetic excitations in YIG, it acquires a phase modulation at the frequency of the excitations, which is analyzed by a six-pass Fabry-Perot interferometer. The resulting signal-the BLS intensity-is proportional to the intensity of the magnetic oscillations at the position of the probing spot. The wavelength of the laser is chosen to be $473 \mathrm{~nm}$, which provides a high sensitivity of the method for measurements with ultra-thin YIG. The power of the probing light is as low as $0.1 \mathrm{~mW}$, which guarantees negligible laserinduced heating of the sample.

We first analyze the effects of SOT on the thermally driven magnetization fluctuations in the YIG waveguide. In these experiments, we only apply the dc current through the Pt layer and record the spectra of thermal magnetic fluctuations for different values of $I$. The representative spectra shown in Figs. 2(a) and 2(b) illustrate the process of the enhancement of magnetic fluctuations by SOT. Note that the enhancement is observed only for positive currents, whereas for negative currents, the fluctuations are suppressed, ${ }^{36}$ in agreement with the symmetry of SHE. In Fig. 2(c), we show the current dependences of the integral intensity in the BLS spectra and its inverse value. In agreement with the theory, ${ }^{37}$
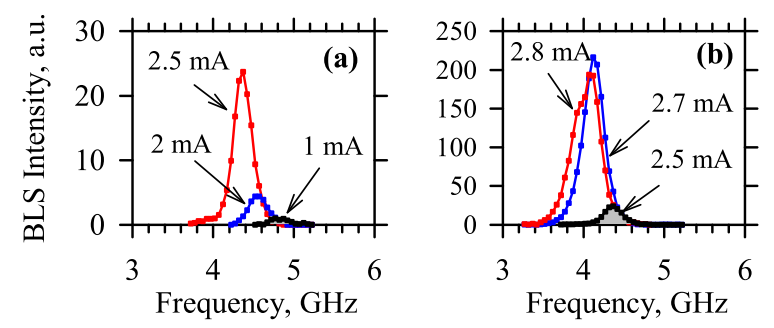

(c)

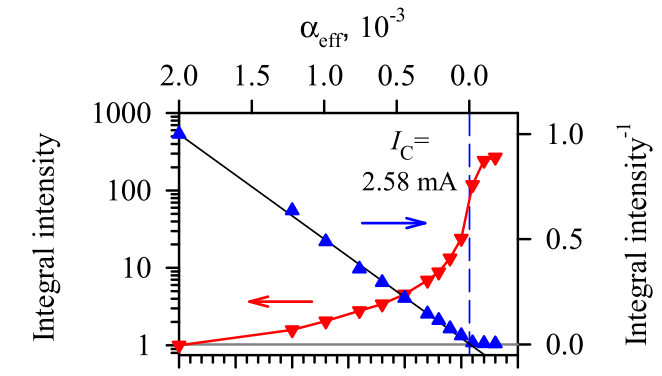

(d)

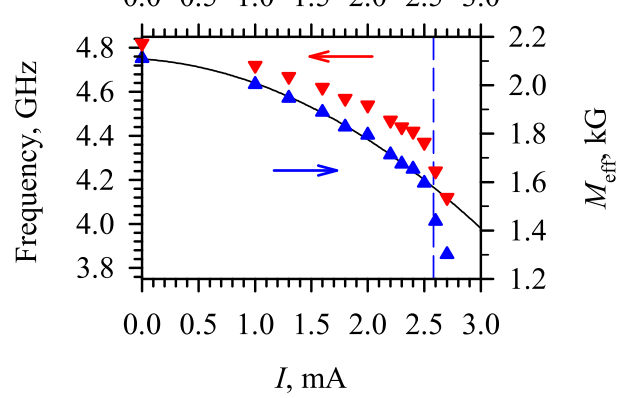

FIG. 2. (a) and (b) Representative BLS spectra of magnetic fluctuations in the YIG waveguide recorded for different dc currents, as labeled. (c) Current dependence of the spectrally integrated BLS intensity and of its inverse value. Vertical dashed line marks the current $I_{\mathrm{C}}$, at which the magnetic damping is completely compensated by the spin-orbit torque. Straight solid line is the linear fit of the experimental data. (d) Current dependence of the frequency of the quasi-uniform ferromagnetic resonance and of the effective magnetization. Solid line is the parabolic fit of the experimental data at $I<I_{\mathrm{C}}$. The data were obtained at $H_{0}=1000 \mathrm{Oe}$.

the latter quantity shows a well-defined linear behavior. By fitting the experimental data by a linear function, we determine the current $I_{\mathrm{C}}=2.58 \mathrm{~mA}$, at which the integral intensity is expected to diverge. This current corresponds to the complete compensation of the damping by SOT. ${ }^{37}$ For $I>I_{\mathrm{C}}$, the system enters the auto-oscillation regime characterized by large amplitudes of the magnetization dynamics (Fig. 2(b)). We note, however, that the auto-oscillation spectra (Fig. 2(b)) are relatively broad, suggesting multimode autooscillations, as expected for a spatially extended system. ${ }^{14,36}$ Based on the parameters of the Pt layer, we estimate the threshold current density of $3 \times 10^{11} \mathrm{~A} / \mathrm{m}^{2}$, which is close to the value obtained for the spin-torque oscillators based on the similar YIG/Pt bilayer. ${ }^{33,34}$ Taking into account the value of the Gilbert damping constant $\alpha=2 \times 10^{-3}$ for $I=0,{ }^{34}$ we additionally show in the top horizontal scale in Fig. 2(c) the values of the effective damping constant corresponding to the applied dc current.

The obtained spectral data allow us to determine the current dependence of the frequency of the quasi-uniform ferromagnetic resonance in the YIG waveguide (down-triangles in Fig. 2(d)) and calculate the corresponding effective magnetization $M_{\text {eff }}$ (up-triangles in Fig. 2(d)). These data show that at $I<I_{\mathrm{C}}, M_{\mathrm{eff}}$ reduces proportional to $I^{2}$ (see parabolic 
fit of the data in Fig. 2(d)), which suggests that the reduction is mainly caused by the Joule heating of YIG by the electrical current in Pt. The current dependence of the effective magnetization exhibits a clear kink at $I=I_{\mathrm{C}}$ and dramatically decreases at larger currents. These behaviors correlate well with the onset of large-amplitude magnetic auto-oscillations causing a strong decrease in the static component of the magnetization vector.

Next, we analyze the effects of SOT on the propagation characteristics of coherent spin waves excited by the microwave current in the antenna. The measurements are performed by applying a microwave signal at the frequency corresponding, for the given conditions, to a spin wave with the wavelength of about $5 \mu \mathrm{m}$, which can be efficiently excited by the used $3 \mu \mathrm{m}$ wide inductive antenna and possess sufficiently large group velocity. ${ }^{35}$ The propagation of spin waves is mapped by rastering the probing laser spot over the surface of the YIG waveguide with the step sizes of 200 and $250 \mathrm{~nm}$ in the transverse and the longitudinal directions, respectively. In Fig. 3(a), we show a representative map of the BLS intensity, proportional to the local intensity in the spin wave, obtained for $I=2.55 \mathrm{~mA}$. As seen from these data, the spin wave propagates along the waveguide nearly uniformly without changing its transverse profile (inset in

(a)

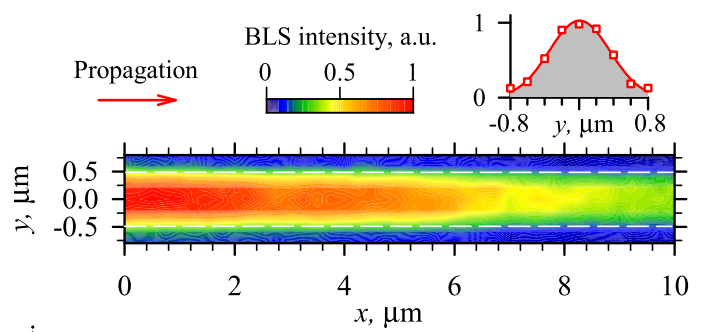

(b)

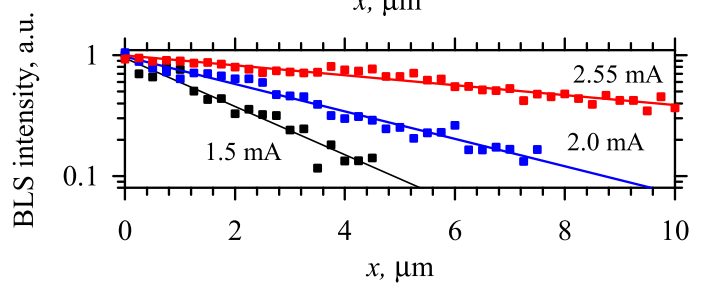

(c)

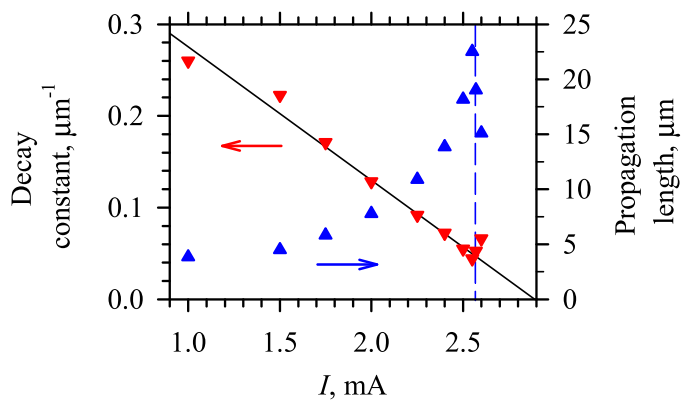

FIG. 3. (a) Normalized spatial intensity map of the propagating spin wave excited by the antenna. The map was recorded for $I=2.55 \mathrm{~mA}$. The mapping was performed by rastering the probing spot over the area $1.6 \times 10 \mu \mathrm{m}$, which is larger than the waveguide width of $1 \mu \mathrm{m}$. Dashed lines show the edges of the waveguide. The inset shows the transverse profile of the spinwave intensity. (b) Dependences of the spin-wave intensity on the propagation coordinate for different currents, as labeled, in the log-linear scale. Lines show the exponential fit of the experimental data. (c) Current dependences of the propagation length and of the decay constant. Vertical dashed line marks $I_{\mathrm{C}}$. Solid line is the linear fit of the experimental data at $I<I_{\mathrm{C}}$. The data were obtained at $H_{0}=1000 \mathrm{Oe}$.
Fig. 3(a)), which is a clear signature of the single-mode propagation regime caused by the strong separation of the transverse modes in a narrow waveguide. ${ }^{35}$ The intensity of the wave decreases by only $60 \%$ over the propagation path of $10 \mu \mathrm{m}$.

To characterize the propagation length of spin waves and its dependence on the current, we plot in Fig. 3(b) the dependences of the spin-wave intensity on the propagation coordinate obtained for different dc currents in the Pt layer. These data show that spin waves in the waveguide experience a well-defined exponential decay (note the logarithmic vertical scale) $\sim \exp (-2 x / \xi)$, where $\xi$ is the propagation length defined as a distance over which the wave amplitude decreases by a factor of $e$. The propagation length strongly increases with the increase in the dc current, as expected for the effect of SOT on the effective magnetic damping.

Figure 3(c) summarizes the results of the spatially resolved measurements. The propagation length (up-triangles) monotonously increases with the increase in $I<I_{\mathrm{C}}$ and then shows an abrupt decrease at $I>I_{\mathrm{C}}$. We note that, according to the data in Fig. 2, in the latter regime, the magnetic damping should be overcompensated by SOT, which is expected to result in a spin-wave amplification. In contradiction to this naive expectation, the propagation length decreases rapidly at $I>I_{\mathrm{C}}$, so that already at $I=2.65 \mathrm{~mA}$ the BLS signal from the coherent spin wave excited by the antenna completely vanishes. This experimental observation can be attributed to the strong nonlinear scattering of coherent spin waves from largeamplitude current-induced magnetic auto-oscillations due to the nonlinear magnon-magnon scattering processes, which are known to be highly efficient in low-damping YIG films. ${ }^{38}$

To characterize the variation of the propagation length with current in detail, we plot in Fig. 3(c) its inverse valuethe decay constant (down-triangles), which is proportional to the effective Gilbert damping constant $\alpha_{\text {eff }}$. In agreement with the simple theoretical model assuming the linear variation of $\alpha_{\text {eff }}$ with current, the decay constant shows a linear dependence on $I$. By extrapolating this dependence to $I=0$, we obtain the propagation length at zero current $\xi_{0}=2.4 \mu \mathrm{m}$. This value reasonably agrees with the theoretical value of $\xi=3.0 \mu \mathrm{m}$ calculated based on the theory of Ref. 35 for spin waves with the wavelength of $5 \mu \mathrm{m}$ propagating in the YIG/ Pt waveguide characterized by the Gilbert damping $\alpha=2 \times 10^{-3}$ (Ref. 34).

Additionally, one expects the linear dependence in Fig. 3 (c) to cross zero at $I=I_{\mathrm{C}}$, which corresponds to an infinitely large propagation length under conditions of the complete damping compensation. The data in Fig. 3(c) show, however, that the linear fit yields the intercept value larger than $I_{\mathrm{C}}$. This disagreement can be attributed to the Joule heating of the waveguide by the electric current in $\mathrm{Pt}$, resulting in the significant reduction of the effective magnetization (Fig. 2(d)). Since the propagation length is proportional to the group velocity, which is known to decrease with the decrease in $M_{\text {eff }}$, the effects of the heating on the propagation length counteract those of SOT and do not allow one to achieve the decay-free propagation regime. We note, however, that the maximum achieved propagation length of $22.5 \mu \mathrm{m}$ is nearly by a factor of 2 larger compared to the value of $12 \mu \mathrm{m}$ 


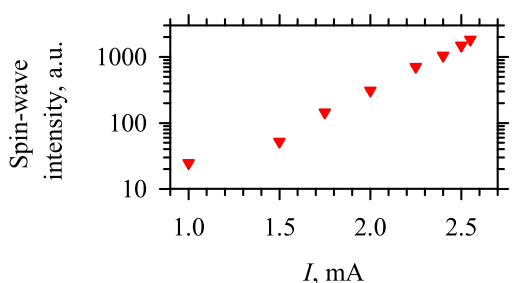

FIG. 4. Current dependence of the intensity of the spin wave at the output of a $10 \mu \mathrm{m}$ long transmission line calculated based on the experimentally measured propagation length. The intensity is normalized by the value at $I=0$.

estimated for a waveguide made of a bare YIG film without Pt on top $\left(\alpha=5 \times 10^{-4}\right)$.

Finally, we discuss the significance of the achieved propagation-length control for the performance characteristics of spin-wave transmission lines. Taking the experimental values of the propagation length $\xi(I)$ (Fig. 3(c)), we calculate the intensity of the spin wave at the output of a transmission line with the length $L=10 \mu \mathrm{m}$ as $J(I)=J_{0} \exp (-2 L / \xi)$, where $J_{0}$ is the input intensity. The results normalized by the value at $I=0$ are shown in Fig. 4. As one clearly sees from these data, the achieved propagation-length control provides the opportunity to increase the intensity of spin waves at the output of a transmission line with technologically relevant length by many orders of magnitude.

In conclusion, we demonstrated that the SOT is a highly efficient mechanism for the control of propagation characteristics of spin waves in ultra-thin-film YIG/Pt systems. We also find that the main limiting factor reducing this efficiency is the reduction of the effective magnetization due to the Joule heating, which can be minimized by the reduction of the waveguide width and/or by integration into the devices of heat-sink elements. Our data also show that the implementation of true amplification of spin waves is hindered by the excitation of large-amplitude auto-oscillations. Further investigations are needed to understand, whether this problem can be overcome. Our observations should stimulate both the development of advanced magnonic devices and theoretical work to deepen the understanding of the interaction of spin currents with spin waves.

We acknowledge E. Jacquet, R. Lebourgeois, R. Bernard, and A. H. Molpeceres for their contribution to sample growth, and O. d'Allivy Kelly and A. Fert for fruitful discussions. This research was partially supported by the Deutsche Forschungsgemeinschaft, the ANR Grant Trinidad (ASTRID 2012 Program), and the program Megagrant No. 14.Z50.31.0025 of the Russian Ministry of Education and Science. M.C. acknowledges DGA for the financial support. V.V.N. acknowledges the support from the Competitive Growth of KFU.

${ }^{1}$ M. I. Dyakonov and V. I. Perel, Sov. Phys. JETP Lett. 13, 467 (1971).

${ }^{2}$ J. E. Hirsch, Phys. Rev. Lett. 83, 1834 (1999).

${ }^{3}$ V. M. Edelstein, Solid State Commun. 73, 233-235 (1990).

${ }^{4}$ A. Chernyshov, M. Overby, X. Liu, J. K. Furdyna, Y. Lyanda-Geller, and L. P. Rokhinson, Nat. Phys. 5, 656-659 (2009).

${ }^{5}$ I. M. Miron, K. Garello, G. Gaudin, P.-J. Zermatten, M. V. Costache, S. Auffret, S. Bandiera, B. Rodmacq, A. Schuhl, and P. Gambardella, Nature 476, 189-93 (2011).

${ }^{6}$ L. Liu, C.-F. Pai, Y. Li, H. W. Tseng, D. C. Ralph, and R. A. Buhrman, Science 336, 555-558 (2012).
${ }^{7}$ Y. Fan, P. Upadhyaya, X. Kou, M. Lang, S. Takei, Z. Wang, J. Tang, L. He, L.-T. Chang, M. Montazeri, G. Yu, W. Jiang, T. Nie, R. N. Schwartz, Y. Tserkovnyak, and K. L. Wang, Nat. Mater. 13, 699-704 (2014).

${ }^{8}$ A. R. Mellnik, J. S. Lee, A. Richardella, J. L. Grab, P. J. Mintun, M. H. Fischer, A. Vaezi, A. Manchon, E.-A. Kim, N. Samarth, and D. C. Ralph, Nature 511, 449-451 (2014).

${ }^{9}$ X. Qiu, K. Narayanapillai, Y. Wu, P. Deorani, D.-H. Yang, W.-S. Noh, J.H. Park, K.-J. Lee, H.-W. Lee, and H. Yang, Nat. Nanotechnol. 10, 333-338 (2015).

${ }^{10}$ V. E. Demidov, S. Urazhdin, H. Ulrichs, V. Tiberkevich, A. Slavin, D. Baither, G. Schmitz, and S. O. Demokritov, Nat. Mater. 11, 1028 (2012).

${ }^{11}$ L. Liu, C.-F. Pai, D. C. Ralph, and R. A. Buhrman, Phys. Rev. Lett. 109, 186602 (2012).

${ }^{12}$ R. H. Liu, W. L. Lim, and S. Urazhdin, Phys. Rev. Lett. 110, 147601 (2013).

${ }^{13}$ V. E. Demidov, S. Urazhdin, A. Zholud, A. V. Sadovnikov, and S. O. Demokritov, Appl. Phys. Lett. 105, 172410 (2014).

${ }^{14}$ Z. Duan, A. Smith, L. Yang, B. Youngblood, J. Lindner, V. E. Demidov, S. O. Demokritov, and I. N. Krivorotov, Nat. Commun. 5, 5616 (2014).

${ }^{15}$ V. E. Demidov, S. Urazhdin, A. B. Rinkevich, G. Reiss, and S. O. Demokritov, Appl. Phys. Lett. 104, 152402 (2014).

${ }^{16}$ K. An, D. R. Birt, C.-F. Pai, K. Olsson, D. C. Ralph, R. A. Buhrman, and X. Li, Phys. Rev. B 89, 140405(R) (2014).

${ }^{17}$ V. V. Kruglyak, S. O. Demokritov, and D. Grundler, J. Phys. D: Appl. Phys. 43, 264001 (2010).

${ }^{18}$ S. Urazhdin, V. E. Demidov, H. Ulrichs, T. Kendziorczyk, T. Kuhn, J. Leuthold, G. Wilde, and S. O. Demokritov, Nat. Nanotechnol. 9, 509-513 (2014).

${ }^{19}$ A. V. Chumak, V. I. Vasyuchka, A. A. Serga, and B. Hillebrands, Nat. Phys. 11, 453-461 (2015).

${ }^{20}$ J. C. Slonczewski, J. Magn. Magn. Mater. 159, L1-L7 (1996).

${ }^{21}$ L. Berger, Phys. Rev. B 54, 9353-9358 (1996).

${ }^{22}$ V. E. Demidov, S. Urazhdin, A. Zholud, A. V. Sadovnikov, A. N. Slavin, and S. O. Demokritov, Sci. Rep. 5, 8578 (2015).

${ }^{23}$ V. E. Demidov, S. Urazhdin, R. Liu, B. Divinskiy, A. Telegin, and S. O. Demokritov, Nat. Commun. 7, 10446 (2016).

${ }^{24}$ Y. Kajiwara, K. Harii, S. Takahashi, J. Ohe, K. Uchida, M. Mizuguchi, H. Umezawa, H. Kawai, K. Ando, K. Takanashi, S. Maekawa, and E. Saitoh, Nature 464, 262-266 (2010).

${ }^{25}$ A. A. Serga, A. V. Chumak, and B. Hillebrands, J. Phys. D: Appl. Phys. 43, 264002 (2010).

${ }^{26}$ Z. Wang, Y. Sun, M. Wu, V. Tiberkevich, and A. Slavin, Phys. Rev. Lett. 107, 146602 (2011).

${ }^{27}$ E. Padron-Hernandez, A. Azevedo, and S. M. Rezende, Appl. Phys. Lett. 99, 192511 (2011).

${ }^{28}$ Y. Sun, Y.-Y. Song, H. Chang, M. Kabatek, M. Jantz, W. Schneider, M. Wu, H. Schultheiss, and A. Hoffmann, Appl. Phys. Lett. 101, 152405 (2012).

${ }^{29}$ O. d'Allivy Kelly, A. Anane, R. Bernard, J. Ben Youssef, C. Hahn, A. H. Molpeceres, C. Carrétéro, E. Jacquet, C. Deranlot, P. Bortolotti, R. Lebourgeois, J.-C. Mage, G. de Loubens, O. Klein, V. Cros, and A. Fert, Appl. Phys. Lett. 103, 082408 (2013).

${ }^{30}$ H. Yu, O. d'Allivy Kelly, V. Cros, R. Bernard, P. Bortolotti, A. Anane, F. Brandl, R. Huber, I. Stasinopoulos, and D. Grundler, Sci. Rep. 4, 6848 (2014).

${ }^{31}$ M. B. Jungfleisch, W. Zhang, W. Jiang, H. Chang, J. Sklenar, S. M. Wu, J. E. Pearson, A. Bhattacharya, J. B. Ketterson, M. Wu, and A. Hoffmann, J. Appl. Phys. 117, 17D128 (2015).

${ }^{32}$ C. Hauser, T. Richter, N. Homonnay, C. Eisenschmidt, M. Qaid, H. Deniz, D. Hesse, M. Sawicki, S. G. Ebbinghaus, and G. Schmidt, Sci. Rep. 6, 20827 (2016).

${ }^{33}$ A. Hamadeh, O. d'Allivy Kelly, C. Hahn, H. Meley, R. Bernard, A. H. Molpeceres, V. V. Naletov, M. Viret, A. Anane, V. Cros, S. O. Demokritov, J. L. Prieto, M. Muñoz, G. de Loubens, and O. Klein, Phys. Rev. Lett. 113, 197203 (2014).

${ }^{34}$ M. Collet, X. de Milly, O. d'Allivy Kelly, V. V. Naletov, R. Bernard, P. Bortolotti, J. Ben Youssef, V. E. Demidov, S. O. Demokritov, J. L. Prieto, M. Munoz, V. Cros, A. Anane, G. de Loubens, and O. Klein, Nat. Commun. 7, 10377 (2016).

${ }^{35}$ V. E. Demidov and S. O. Demokritov, IEEE Trans. Magn. 51, 0800215 (2015).

${ }^{36}$ V. E. Demidov, S. Urazhdin, E. R. J. Edwards, M. D. Stiles, R. D. McMichael, and S. O. Demokritov, Phys. Rev. Lett. 107, 107204 (2011).

${ }^{37}$ A. Slavin and V. Tiberkevich, IEEE Trans. Magn. 45, 1875 (2009).

${ }^{38}$ A. G. Gurevich and G. A. Melkov, Magnetization Oscillations and Waves (CRC, New York, 1996). 